\title{
Independence of HIF1a and androgen signaling pathways in prostate cancer
}

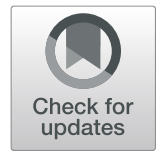

Maxine G. B. Tran ${ }^{1,2+}$, Becky A. S. Bibby ${ }^{3 \dagger}$, Lingjian Yang ${ }^{3}$, Franklin Lo ${ }^{1}$, Anne Y. Warren ${ }^{4}$, Deepa Shukla ${ }^{5}$, Michelle Osborne ${ }^{1}$, James Hadfield ${ }^{1}$, Thomas Carroll', Rory Stark', Helen Scott ${ }^{1}$, Antonio Ramos-Montoya', Charlie Massie $^{1,6}$, Patrick Maxwell7 , Catharine M. L. West ${ }^{3,8}$, lan G. Mills $s^{9,10^{* *}}$ (1) and David E. Neal ${ }^{10,11+}$

\begin{abstract}
Background: Therapeutic targeting of the androgen signaling pathway is a mainstay treatment for prostate cancer. Although initially effective, resistance to androgen targeted therapies develops followed by disease progression to castrate-resistant prostate cancer (CRPC). Hypoxia and HIF1a have been implicated in the development of resistance to androgen targeted therapies and progression to CRCP. The interplay between the androgen and hypoxia/HIF1a signaling axes was investigated.

Methods: In vitro stable expression of HIF1a was established in the LNCaP cell line by physiological induction or retroviral transduction. Tumor xenografts with stable expression of HIF1a were established in castrated and noncastrated mouse models. Gene expression analysis identified transcriptional changes in response to androgen treatment, hypoxia and HIF1a. The binding sites of the AR and HIF transcription factors were identified using ChIPseq.

Results: Androgen and HIF1a signaling promoted proliferation in vitro and enhanced tumor growth in vivo. The stable expression of HIF1a in vivo restored tumor growth in the absence of endogenous androgens. Hypoxia reduced AR binding sites whereas HIF binding sites were increased with androgen treatment under hypoxia. Gene expression analysis identified seven genes that were upregulated both by AR and HIF1a, of which six were prognostic.

Conclusions: The oncogenic AR, hypoxia and HIF1a pathways support prostate cancer development through independent signaling pathways and transcriptomic profiles. AR and hypoxia/HIF1a signaling pathways independently promote prostate cancer progression and therapeutic targeting of both pathways simultaneously is warranted.
\end{abstract}

Keywords: Prostate cancer, Androgen signaling, HIF1a signaling, Hypoxia

\footnotetext{
* Correspondence: ian.mills@nds.ox.ac.uk

${ }^{+}$Maxine G. B. Tran, Becky A. S. Bibby, lan G. Mills and David E. Neal contributed equally to this work.

${ }^{9}$ Patrick G Johnston Centre for Cancer Research and Cell Biology, Queens University Belfast, Belfast BT9 7AE, UK

${ }^{10}$ Nuffield Department of Surgical Sciences, University of Oxford, Oxford OX3 9DU, UK

Full list of author information is available at the end of the article
}

(c) The Author(s). 2020 Open Access This article is licensed under a Creative Commons Attribution 4.0 International License, which permits use, sharing, adaptation, distribution and reproduction in any medium or format, as long as you give appropriate credit to the original author(s) and the source, provide a link to the Creative Commons licence, and indicate if changes were made. The images or other third party material in this article are included in the article's Creative Commons licence, unless indicated otherwise in a credit line to the material. If material is not included in the article's Creative Commons licence and your intended use is not permitted by statutory regulation or exceeds the permitted use, you will need to obtain permission directly from the copyright holder. To view a copy of this licence, visit http://creativecommons.org/licenses/by/4.0/. The Creative Commons Public Domain Dedication waiver (http://creativecommons.org/publicdomain/zero/1.0/) applies to the data made available in this article, unless otherwise stated in a credit line to the data. 


\section{Background}

Androgen signaling drives prostate cancer development and progression. Endogenous androgens, testosterone and dihydrotestosterone, bind to the intracellular androgen receptor (AR) which translocates to the nucleus. The AR functions as a transcription factor and activates downstream signaling pathways associated with proliferation, invasion and metabolism [1]. Androgen deprivation therapy (ADT) inhibits AR signaling by blocking the production of androgens or by inhibiting androgen binding to the AR. ADT is used to treat localized, locally advanced and metastatic disease and an estimated $50 \%$ of prostate cancer patients receive ADT [2]. Although ADT is initially effective, resistance subsequently develops and the AR signaling pathway remains active even in the absence of endogenous androgens. The development of androgen independent or castrateresistant prostate cancer (CRPC) is associated with the presence of metastases and a rapid clinical demise [3]. Identifying which patients will progress to CRPC is a major challenge in the treatment of prostate cancer. Understanding the biology that underpins progression to CRPC will support the development of novel strategies to identify, prevent and treat CRPC.

AR activity during CRPC can be sustained through a number of alterations in the expression of the AR, in signaling pathways and in the expression and activity of other transcription factors which can interact with the AR [4]. One of the earliest examples of these changes was the identification of significant X chromosome copy number amplification encompassing the AR gene locus in a significant percentage of CRPC cases [5]. This was associated with AR overexpression and believed to contribute to androgen hypersensitivity. More recently AR splice variants have been reported to be expressed in CRPC which lack the ligand binding domain and display constitutive transcriptional activity and regulate the expression of an altered repertoire of target genes [6]. Researchers working on signaling pathways have identified AR phosphorylation sites for Akt and other important kinases functional downstream of receptor tyrosine kinases [7]. These sites are also thought to maintain AR transcriptional activity under conditions of androgen deprivation. The AR has also been shown by us and others to interact with a range of other transcription factors capable of recruiting into chromatin-associated transcriptional complexes under conditions of androgen deprivation. These include a number of ETS family transcription factors amongst which we have identified ETS1 [8] and GABPA [9] and others have reported interactions with ERG and ETV1 [10-12]. Such interactions are believed to alter the identity of AR binding sites across the genome and consequently also the identity of genes that are regulated by AR recruitment to the genome. It is possible that this is also the basis for effects of the hypoxia inducible factor (HIF) pathway on the AR [13]. HIF is a heterodimer, consisting of a constitutively stable HIF1b and a tightly regulated HIF1a subunit. Under oxygenated conditions, the HIF1a protein is ubiquitinated and rapidly degraded. In the absence of oxygen, HIF1a is stabilized and dimerizes with HIF1b subunits to form an active HIF transcription complex. HIF translocates to the nucleus and induces the expression of genes associated with metabolism, angiogenesis, invasion and cell survival. Hypoxia-independent stabilization can also occur- a condition referred to as pseudohypoxia [14] [15]. Expression of HIF is associated with increased risk and a poor prognosis in prostate cancer $[16,17]$.

Crosstalk between the AR and hypoxia/HIF has been reported. ADT in hypoxia promotes adaptive androgen/ AR-independence, and confers resistance to androgen/ AR-targeted therapy [18] . Co-immunoprecipitation assays have confirmed a direct interaction between AR and HIF1a, and ChIP analysis showed HIF1a interacts with the AR on the PSA gene promoter [19]. Hypoxia induced activation of HIF can also increase expression of the AR [20, 21]. As AR and HIF signaling pathways are major signaling hubs and oncogenic drivers of prostate cancer progression, this study aimed to investigate further the relationship between them. Here we report for the first time that combined AR and HIF1a signaling in vivo promotes tumor growth and demonstrate the capacity of HIF1a to promote tumor growth in the absence of endogenous androgen in vivo. We also show that the AR and HIF/hypoxia signaling pathways function independently regulating the transcription of different subsets of genes with few shared targets.

\section{Methods \\ Cell culture}

LNCaP, LNCaP-Bic, LNCap-OHF and PC3 cell lines (and the corresponding stable transfectants) were cultured in RPMI with glutamine and $10 \%$ fetal calf serum. LNCaP and PC3 cell-lines were procured from the ATCC repository. $\mathrm{LNCaP}-\mathrm{Bic}$ and $\mathrm{LNCaP}-\mathrm{OHF}$ celllines were derived through long term culture in bicalutamide and hydroxyflutamide and were provided by Prof. Zoran Culig (Medical University Innsbruck, Austria). For hypoxia experiments, cells were exposed to $1 \%$ oxygen using either a hypoxic workstation (INVIVO2, Ruskinn, Leeds, UK) or a hypoxic incubator. For AR signaling experiments, LNCaP cells were grown in charcoal stripped serum for $96 \mathrm{~h}$ prior to adding synthetic androgen (R1881) or vehicle control (ethanol). All celllines were authenticated by STR Profiling using the ATCC Authentication Service and were subjected to PCR-based mycoplasma testing every 2 weeks throughout the course of the study with negative results 
throughout. Ethics approval was not required for the derivation and propagation of any of the cell-lines used in this study.

\section{Infection of HIF1a retroviral vectors}

A model of pseudohypoxia was established in androgensensitive LNCaP cells by viral transfection of a vector encoding HIF1a with two amino acid substitutions which prevented its degradation in the presence of oxygen. Viral supernatants were prepared by transfecting the Phoenix packaging cell line (Orbigen, San Diego, CA) using Lipofectamine 2000 (Life Technologies, Paisley, UK). After initial transfection, Phoenix cells were grown at $32^{\circ} \mathrm{C}$. The supernatant was collected and filtered $(0.45 \mu \mathrm{m})$, then supplemented with a 1:4 volume of fresh medium with $7.5 \mu \mathrm{g} / \mathrm{mL}$ Polybrene (Sigma, Poole, UK), and added to LNCaP cells plated on p100 dishes at 30$40 \%$ confluence. After $20 \mathrm{~h}$, cells were washed, and fresh media added for $20 \mathrm{~h}$ before a second round of transfection and G418 selection. The constitutively active form of HIF1a (carrying two substitutions: P402A and P564A) was cloned into pBMN-I-EGFP.

\section{Western blot analysis}

Cell lysis involved urea-SDS buffer supplemented with phenylmethylsulfonyl fluoride (PMSF) as previously described [1]. Immunoblots were visualized with enhanced chemiluminescence reagent or enhanced chemiluminescence plus reagent (Amersham, Arlington Heights, IL). Antibodies used were HIF1a (clone 54, Transduction Labs, Lexington $\mathrm{KY}$ ) and $\alpha$ tubulin (CRUK).

\section{Xenograft experiments}

Xenograft tumors were generated with LNCaP/Empty and LNCaP/HIF1-clone 1 cells that stably expressed a fusion protein of luciferase and yellow fluorescent protein (YFP). There were four groups (Full/non-castrated + LNCaP/Empty, Full/non-castrated + LNCaP/HIF1a clone 1, Castrated + LNCaP/Empty and Castrated + LNCaP/HIF1a clone 1), each consisting of five mice. Tumor growth was monitored weekly through bioluminescence with an IVIS camera (Xenogen) [1].

\section{Clinical material and immunohistochemistry}

Clinical samples were collected from Cambridge University Hospitals NHS Trust as part of the PROMPT study, and ethical approval was granted by the local research and ethics committee (LREC number: $02 / 281 \mathrm{M}$ ) and by the multicenter research and ethics committee (MREC number 01/4061). A tissue microarray (TMA) was constructed, consisting of at least two tumor cores with matched benign prostate tissue cores from each of 41 patients with CRPC. Sections $3 \mu \mathrm{m}$ thick were mounted on Snowcoat X-tra slides (Surgipath, Richmond, IL), dewaxed in xylene and rehydrated using graded ethanol washes. For antigen retrieval, sections were immersed in preheated DAKO target retrieval solution and treated for $90 \mathrm{~s}$ in a pressure cooker (DAKO, Glostrup, Denmark). Antigen/antibody complexes were detected using the DAKO catalyzed signal amplification (CSA) system according to the manufacturer's instructions. Sections were counterstained with hematoxylin for $30 \mathrm{~s}$, dehydrated in graded ethanol washes and mounted (Lamb, London, UK). A rabbit pAb was used for HIF1a immunohistochemistry in the xenograft tumors (\#NB 100-479, Novus Biologicals, Oxford, UK) and a mouse mAb was used for HIF1a immunohistochemistry in the CR-TMA (H1 $\alpha 67$ \# NB 100-105, Novus Biologicals). Immunohistochemistry staining was scored by two independent blinded assessors as 1 (negative), $2(<25 \%$ of nuclei staining), 3 (25-50\% of nuclei staining), 4 (majority of cells - weak staining), 5 (majority of cells - moderate staining) and 6 (majority of cells - strong staining).

\section{Illumina HumanWG v2 BeadArray data analysis}

LNCaP, LNCaP/Empty and LNCaP/HIF1a clone 1 cells were grown in charcoal stripped serum for $96 \mathrm{~h}$ prior to adding $1 \mathrm{nM} \mathrm{R} 1881$ or $0.01 \%$ ethanol (vehicle control) for $4 \mathrm{~h}$ and extracting RNA. Total RNA was extracted using Trizol and isopropanol precipitation, according to the manufacturers' instructions. Quality control was performed with an Agilent Bioanalyser. cRNA was generated and biotin labelled using the Illumina TotalPrep RNA Amplification Kit, according to the manufacturers' instructions. $250 \mathrm{ng}$ of RNA was hybridized per sample in accordance with the manufacturers' instructions and the literature [22]. Scanning was performed using Standard Illumina protocols.

For hypoxia experiments, LNCaP cells were exposed to $1 \%$ hypoxia or normoxia for $24 \mathrm{~h}$ prior to RNA extraction. Gene expression data were generated using the Illumina HumanWGv2 BeadArrays. After background correction, normalization and $\log 2$ transformation, differential expression analysis was performed with LIMMA on probe set level. Probe sets with bad and no match probe scores were omitted from analysis. False discovery rate adjusted $P$ value of 0.05 and 1.5 fold change were applied as cut-off.

\section{Chromatin Immunoprecipitation}

ChIP and re-ChIP was performed as previously described $[8,23]$. Cells were cultured in phenol red-free RPMI media supplemented with $10 \%$ charcoal dextranstripped FBS for $72 \mathrm{~h}$ before adding $1 \mathrm{nM}$ R1881 or $0.01 \%$ ethanol for $4 \mathrm{~h}$. For hypoxic experiments, cells were placed in a hypoxic incubator at $1 \%$ oxygen for 12 $\mathrm{h}$ prior to adding R1881 or ethanol. AR (AR N20, Sc816X, Santa Cruz), HIF1a (ab2185, Abcam), H3K4me1 
(pAb 194-050 Diagenode) and H3K4me3 (pAb 003050, Diagenode, Seraing, Belgium) antibodies were used in the assay. ChIP enrichment was tested by real-time PCR and the remainder was used for single-end SOLEXA library preparation.

\section{ChIP-seq SOLEXA library preparation}

Single-end SOLEXA sequencing libraries were prepared as previously described [23]. Sequence reads were generated using an Illumina Genome Analyzer II and mapped to the reference human genome before peak calling. Called peaks were analysed in $\mathrm{R}$ using ChIPpeakAnno package [24].

\section{Data deposition}

Microarray and ChIP-seq data generated have been deposited within the National Center for Biotechnology Information (NCBI) Gene Expression Omnibus (GEO) database (https://www.ncbi.nlm.nih.gov/geo/) under GSE114734.

Patient cohorts, endpoints and statistical analysis.

Five prostate cancer gene expression cohorts with publically available patient survival data were used to evaluate the prognostic significance of selected genes: TCGA [25], Taylor et al. GSE21032 [26], Long et al. GSE54460
[27], Ross-Adams et al. GSE70770 [25] and Sboner et al. GSE16560 [28] (Supplementary Table 1). For TCGA, GSE54460, GSE70770, GSE16560 gene expression data were downloaded directly. For GSE21032, raw CEL files were downloaded and processed using aroma package.

Biochemical recurrence-free (BCR) survival was the primary endpoint, except for Sboner where only overall survival was available. Patients were stratified into high and low risk groups based on cohort median expression of the gene of interest. Survival estimates were performed using the Kaplan-Meier method. The log-rank test was used to test the null hypothesis of equality of survival distributions. Hazard ratios (HR) and 95\% confidence intervals $(\mathrm{CI})$ were obtained using the Cox proportional hazard model.

\section{Results}

HIF1a expression promotes proliferation and resistance to $A D T$ in vitro and in vivo

The stable overexpression of HIF1a in LNCaP/HIF1a clone 1 and clone 2 (Supplementary Fig. 1A) cells increased proliferation and resistance to ADT (bicalutamide) in vitro (Fig. 1). Growth rate decreased in response to $\mathrm{ADT}$ in $\mathrm{LNCaP} /$ Empty but not $\mathrm{LNCaP} /$

(A)
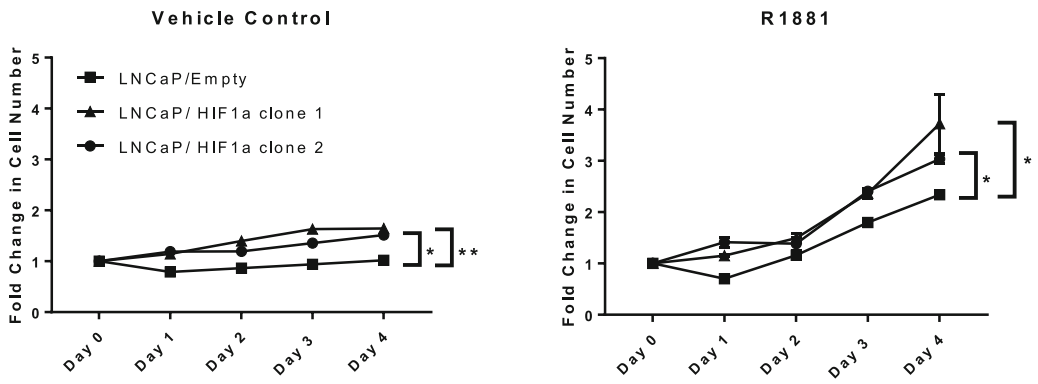

(B)
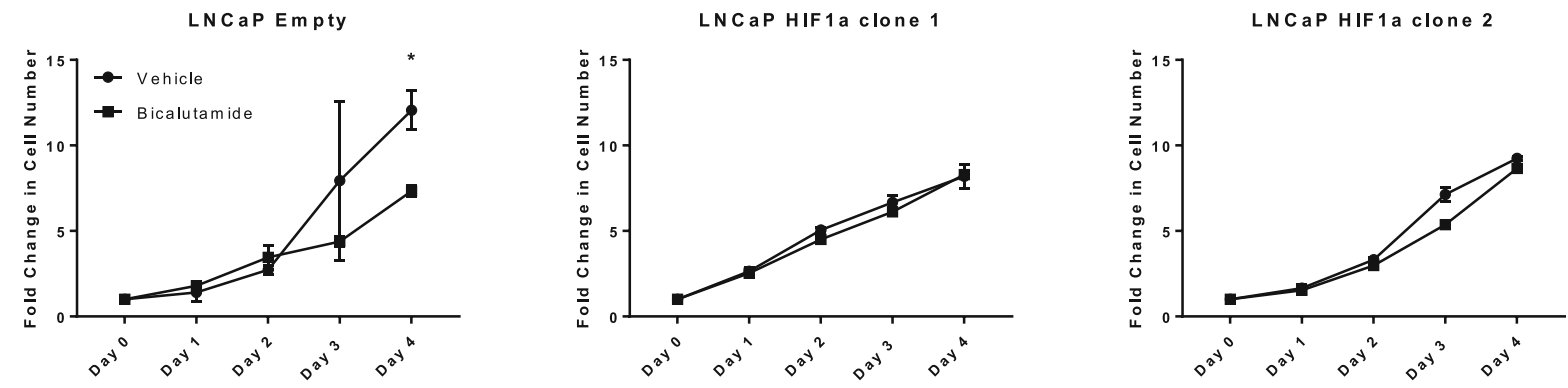

Fig. 1 HIFla overexpression in the androgen dependent LNCaP cell line increased proliferation and resistance to androgen deprivation therapy. a, stable HIF1a expression increased cell proliferation compared to the LNCaP/Empty control cells when cells were treated with the ethanol vehicle control or synthetic androgen R1881 (two way multiple comparison ANOVA; ${ }^{*} p<0.05$, ${ }^{* *} p<0.01$ ). b, stable HIF1a expression led to resistance to bicalutamide treatment (two-tail t-test, ${ }^{*} p=0.058$ ). Data points represent the mean of three intra-assay and two biological repeats \pm SEM. Differences in growth rates are due to cells grown in media containing charcoal stripped serum (androgen depleted media) for $96 \mathrm{~h}$ prior to treatment (a) or in standard RPMI median with FBS that contains androgen and growth stimulants (b) 
HIF1a cells. HIF1a expression was also detected in normoxia in ADT resistant (LNCaP-Bic, LNCaP-OHF) and androgen-independent (PC3) cells but not in androgen sensitive (LNCaP) cells (Supplementary Fig. 1). LNCaP/ HIF1a xenografts grew faster than the LNCaP/Empty tumors, and were resistant to ADT (castrated mouse model; Fig. 2).

\section{Castrate resistant prostate cancers have high HIF1a expression}

HIF1a immunohistochemistry showed high levels of HIF1a expression in CRPC. All CRPC biopsies expressed HIF1a in comparison with only $57 \%$ of benign tumors. Sixty-eight percent of CRPC biopsies had strong HIF1a staining compared with just $8 \%$ of benign tissue (Supplementary Fig. 2).

\section{Only seven genes upregulated by both androgen and} HIF1a

In LNCaP cells, gene expression analysis identified 336 genes upregulated in response to synthetic androgen (Supplementary Table 2) and 579 genes in response to hypoxia (Supplementary Table 3). Forty-seven genes were upregulated by both androgen and hypoxia (Fig. 3a).
There were 149 genes upregulated in response to synthetic androgen in LNCaP/Empty cells and 56 genes upregulated in response to stable HIF1a expression (Fig. 3b). Only seven genes (TWIST1, KCNN2, PPFIBP2, JAG1, SPRED1, IGFBP3, NDRG1) were upregulated by both androgen and HIFla (Fig. 3b). Three genes (SPRED1, IGFBP3, NDRG1) were upregulated by androgen, hypoxia and stable HIF1a expression.

\section{AR and HIF transcription factor binding sites increase differently in response to androgen and hypoxia, respectively}

ChIP-seq analysis identified global AR and HIF DNA binding sites in LNCaP cells exposed to synthetic androgen under normoxia and hypoxia. There were more AR (called peaks range 18,404 to 70,064) compared to HIF (range 523 to 5795) transcription factor binding sites (Table 1). The number of AR binding sites increased with androgen treatment and decreased in hypoxia. However, while hypoxia almost halved the number of AR binding sites, they increased when androgen was added under hypoxia (from 18,404 to 45,635) suggesting an interplay between hypoxia and androgen signalling at the level of AR recruitment to chromatin. AR binding

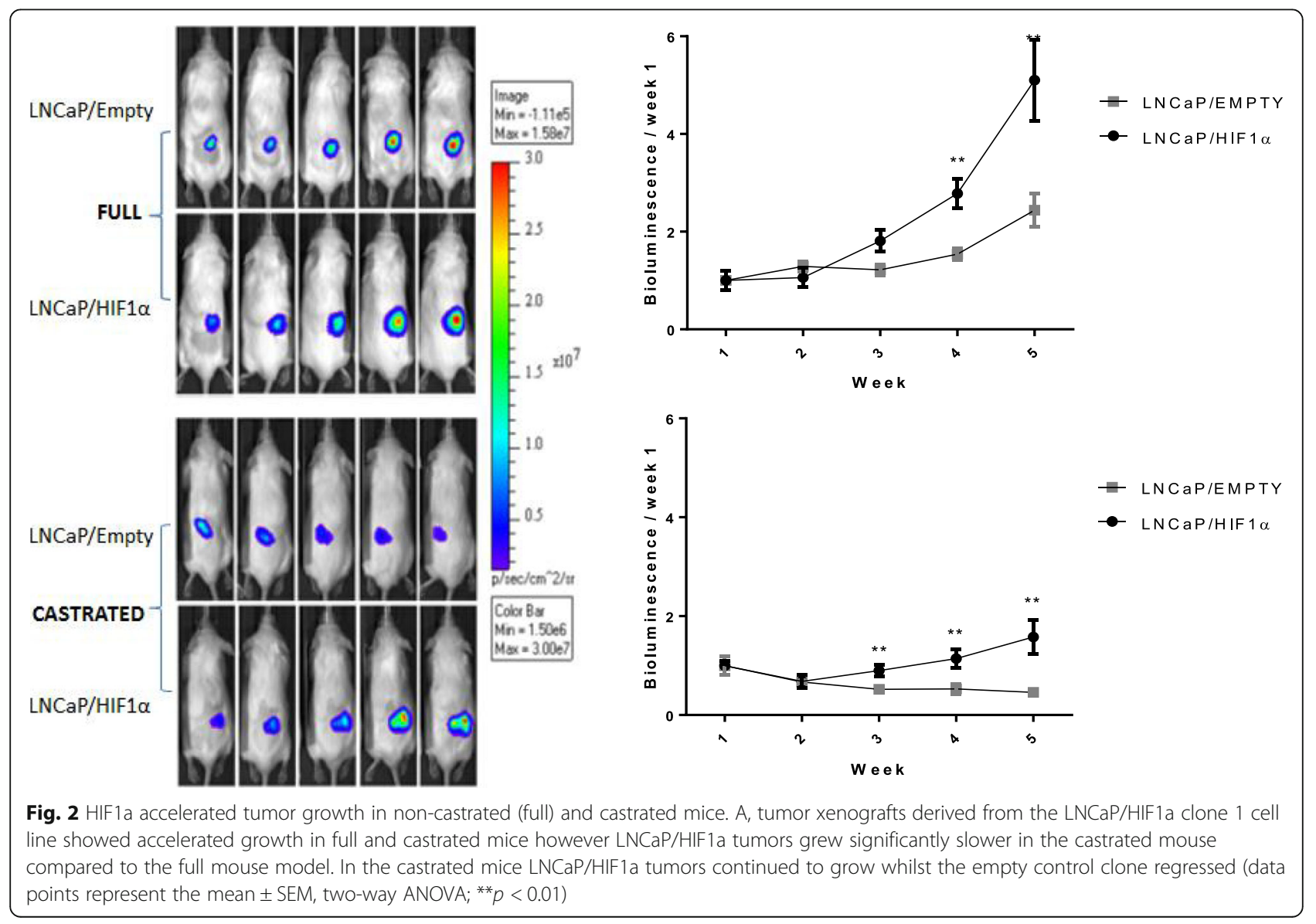




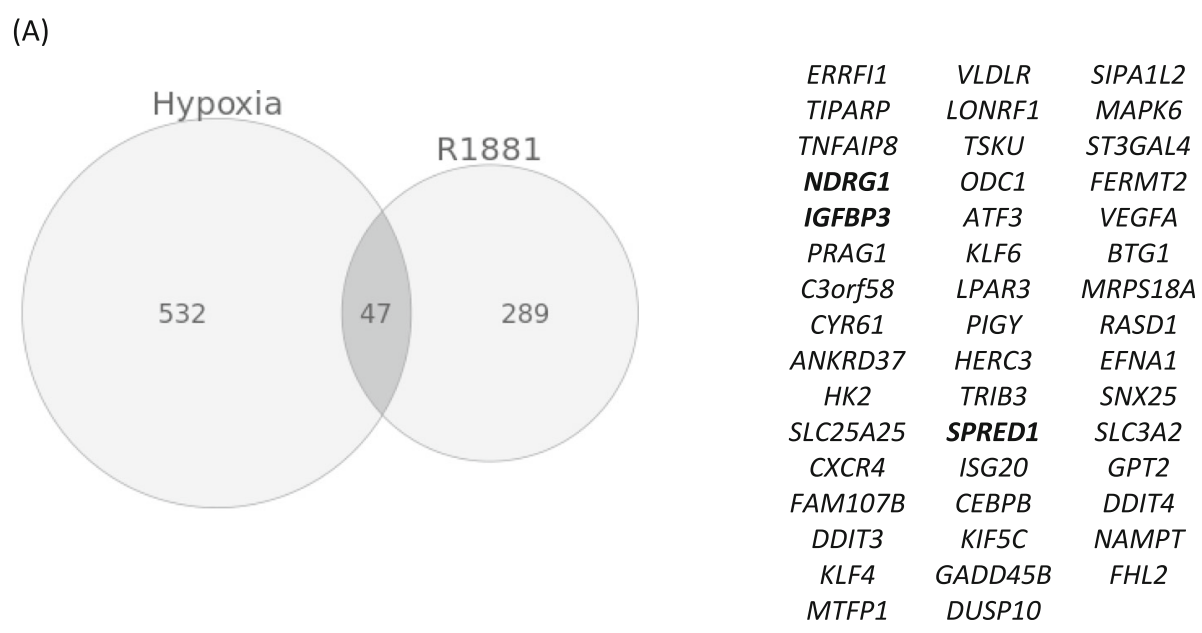

(B)

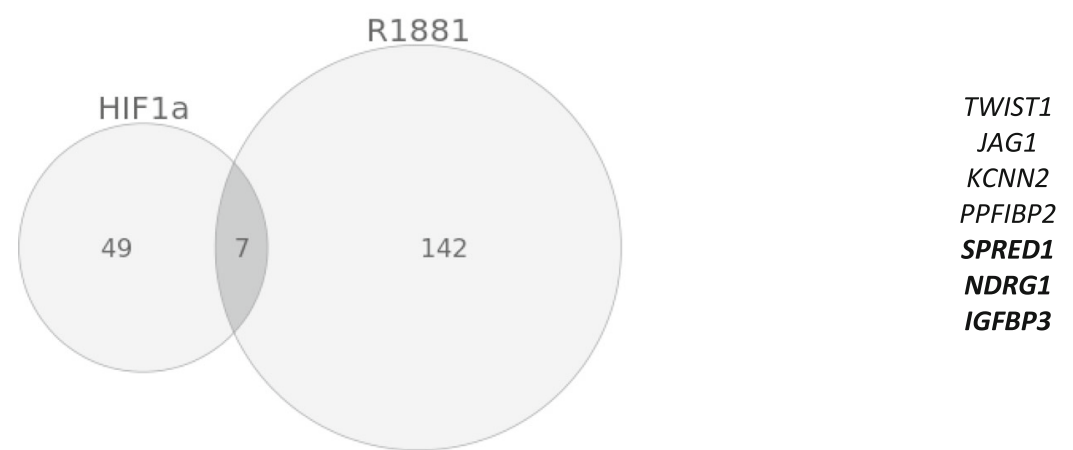

Fig. 3 Genes upregulated by androgen (R1881), hypoxia and HIF1a in LNCaP cells. a, 47 genes upregulated by androgen (LNCaP vehicle control vs. LNCaP R1881, right circle) were independently upregulated by hypoxia (LNCaP normoxia vs. LNCaP 1\% hypoxia, left circle). b, 7 genes upregulated by HIF1a overexpression (LNCaP Empty vs. LNCaP HIF1a, left circle) were also independently upregulated by androgen (LNCaP Empty vehicle control vs. LNCaP Empty R1881, right circle). Three genes were independently upregulated by and androgen, hypoxia and HIF1a (SPRED1, NDRG1 and IGFBP)

sites were highly conserved between the vehicle control and androgen treated cells exposed to normoxia (86\%) and hypoxia (79\%) (Fig. 4a). As expected hypoxia increased the number of HIF binding sites, the greatest number of HIF binding sites was observed in cell treated with combined hypoxia and androgen treatment (Table I). HIF binding sites were not conserved in cells between normoxia and hypoxia in the absence $(6 \%)$ or presence (3\%) of androgen (Fig. 4b). These experiments show androgen increases the number of AR binding sites while conserving those present in the absence of androgen, i.e. AR binds to additional sites. In contrast, hypoxia increases the number of HIF binding sites but with little conservation, i.e. HIF binds to different sites in hypoxia. These observations highlight differences in the effect on transcription when the AR responds to androgen and HIF to hypoxia.

Not all binding sites reflect sites of transcriptional activity. A comparison with histone marks can refine this landscape and help to identify those sites most likely to be active: H3K4me1 is enriched at active and primed enhancers and H3K4me3 in a promoter (i.e. most likely to be active) and stable H3K4me3 has been associated with transcription initiation $[29,30]$. Table 1 shows androgen globally decreased both histone markers. In comparison, hypoxia globally increased the number of histone marker binding sites. Figure 5 A-D shows no change in the distribution of H3K4me1 in response or androgen or hypoxia. Figure $5 \mathrm{E}-\mathrm{H}$ shows an increase in the percentage of H3K4me3 located within promoter regions in response to androgen and a decrease in response to hypoxia. These observations are consistent with androgen increasing and hypoxia decreasing transcription initiation at transcriptional start sites.

The locations of the transcription factors (AR, HIF) and the histone markers (H3K4me1, and H3K4me3) were analysed within the exons and introns of the seven genes identified in the gene expression analysis (Supplementary Fig. 3). Neither AR nor HIF bound within the TWIST1 and IGFBP3 genes (data not shown). There 
Table 1 ChIP-seq global called peaks for AR, HIF and histone methylation markers

\begin{tabular}{lll}
\hline Antibody & Condition & Called peaks \\
\hline AR & R1881/Normoxia & 70,064 \\
AR & R1881/Hypoxia & 45,635 \\
AR & Ethanol/Normoxia & 35,320 \\
AR & Ethanol/Hypoxia & 18,404 \\
HIF & R1881/Normoxia & 523 \\
HIF & R1881/Hypoxia & 5795 \\
HIF & Ethanol/Normoxia & 1181 \\
HIF & Ethanol/Hypoxia & 1746 \\
H3K4Me1 & R1881/Normoxia & 74,269 \\
H3K4Me1 & R1881/Hypoxia & 79,415 \\
H3K4Me1 & Ethanol/Normoxia & 90,818 \\
H3K4Me1 & Ethanol/Hypoxia & 95,424 \\
H3K4Me3 & R1881/Normoxia & 16,419 \\
H3K4Me3 & R1881/Hypoxia & 18,627 \\
H3K4Me3 & Ethanol/Normoxia & 24,358 \\
H3K4Me3 & Ethanol/Hypoxia & 38,929 \\
\hline
\end{tabular}

were more $\mathrm{AR}$, HIF, H3K4me1 and H3K4me3 binding sites in KCNN2 and PPFIBP2 compared to the other genes (Table 2). These observations suggest that KCNN2 and PPFIBP2 are directly regulated by promoter proximal and intragenic recruitment of the AR and HIF1 whereas TWIST1 and IGFBP3 may be enhancer regulated. Indeed changes in IGFBP3 expression have been shown to be affected by and to affect the expression of a range of genes through long-range chromatin and interchromosomal interactions [31]. In addition, TWIST1 is known to function as a transcriptional driver of EMT. Consequently, although the number of genes we have identified as co-ordinately regulated by the AR and HIF1 is small in number their impact may be farreaching.

\section{Effect of TWIST1, KCNN2, PPFIBP2, JAG1, SPRED1, IGFBP3 and NDRG1 on prognosis}

Five publically available prostatectomy gene expression cohorts were used to test the prognostic significance of the seven genes upregulated by androgen, stable HIF1a expression and hypoxia (Table 3). TWIST1 was the most prognostic with high expression associated with poor a prognosis in three cohorts. Five of the genes were prognostic in a single cohort and SPRED1 had no prognostic significance (Table 3). We further compared TWIST1 to a recently published hypoxia-gene associated prognostic signature for prostate cancer [32]. The 28-gene prognostic signature was derived from the TCGA cohort, and had a significant proportion of genes absent in Sboner et al. cohort. In Taylor et al. both TWIST1 (HR 2.45, 95\% CI 1.01-5.93, $P=0.047$ ) and the 28-gene signature (HR 4.48, 95\% CI 1.67-12.04, $P=0.0030$ ) retained prognostic significance.

\section{Discussion}

Hypoxia and HIF1a signaling are widely regarded as cause and consequence, but there is increasing evidence of pseudohypoxia - the expression of HIF1a in normoxia - in multiple cancers [33]. Our LNCaP/HIF1a clones represent a model of pseudohypoxia. Stable HIF1a

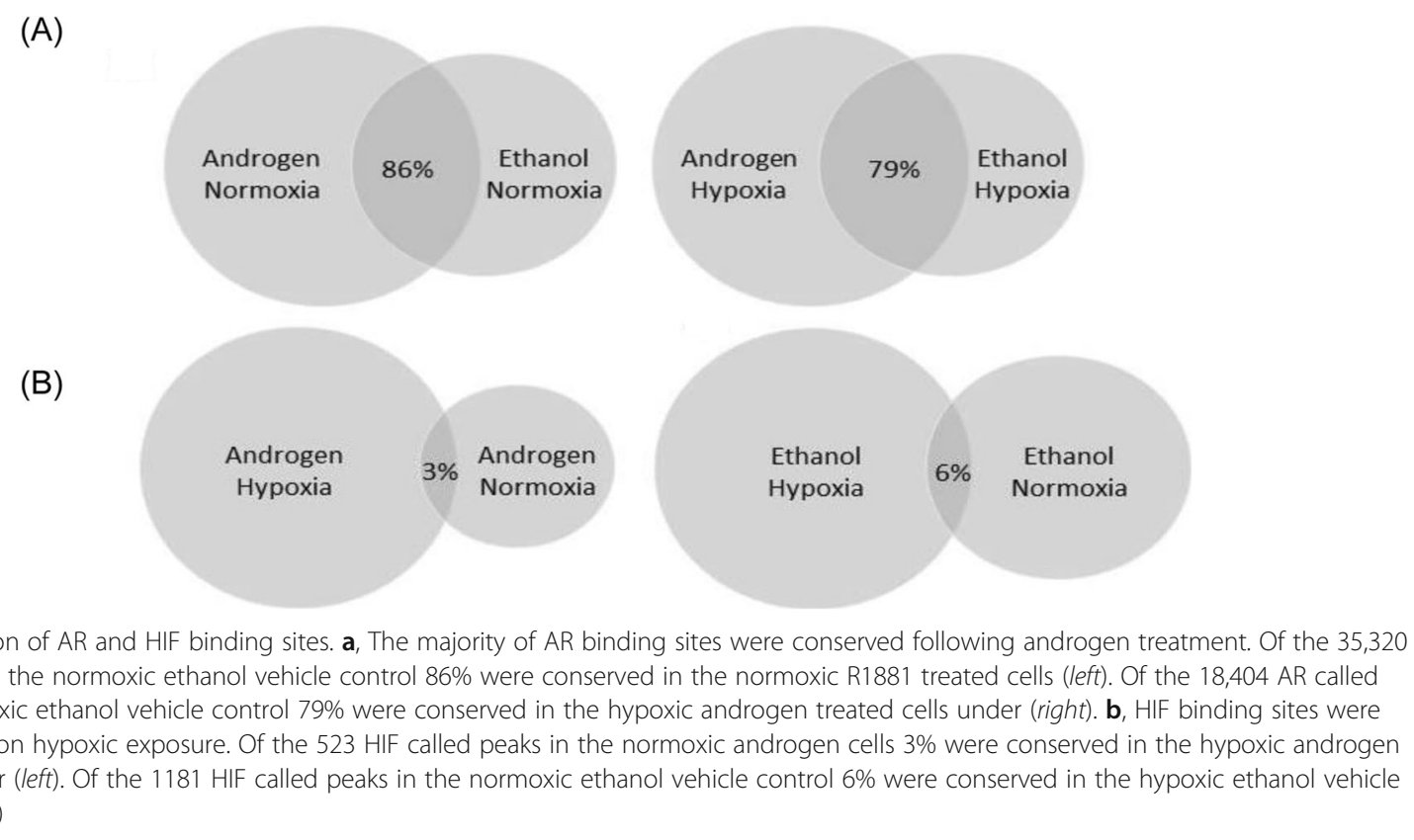




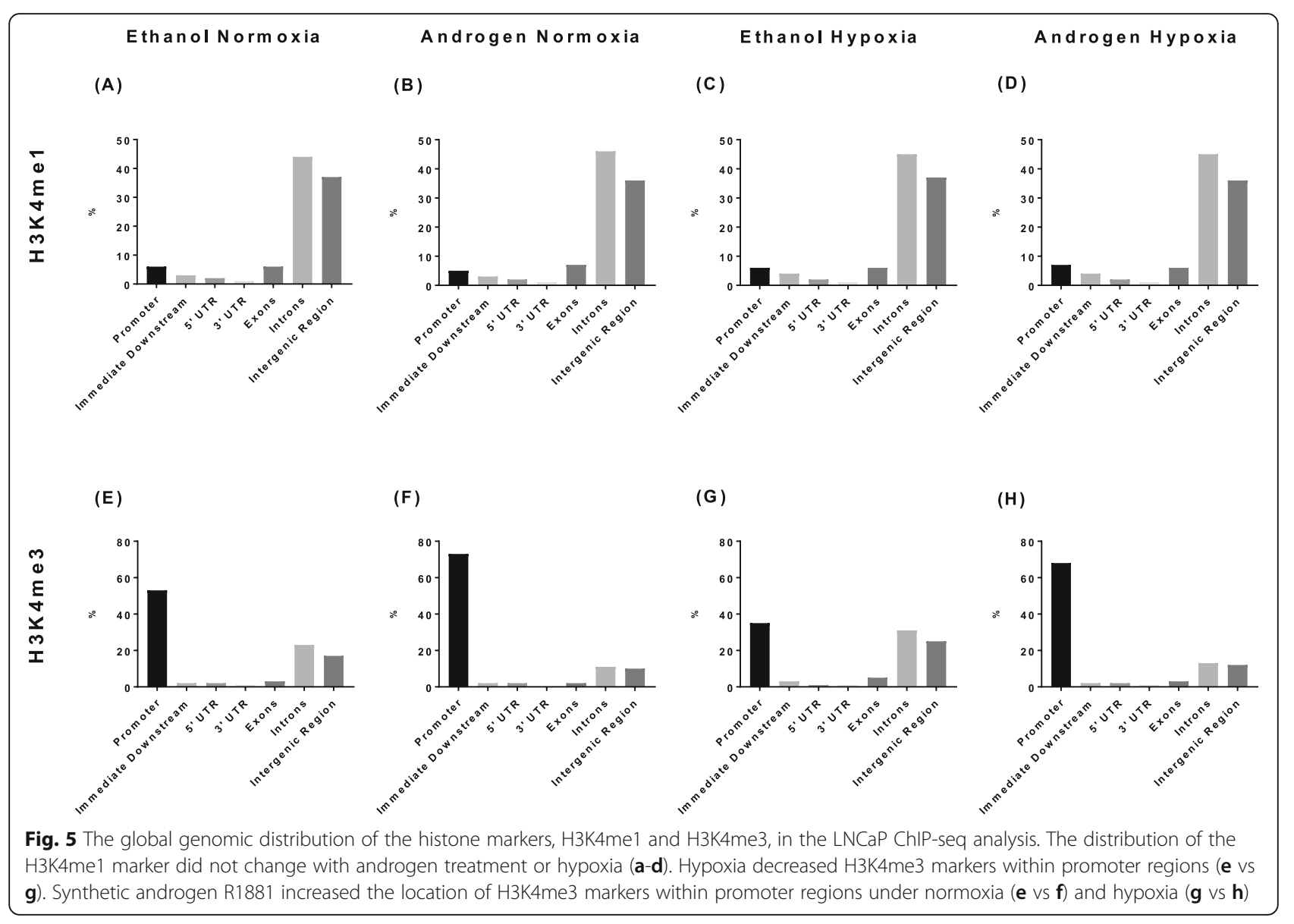

Table 2 Numbers of binding sites of transcription factors and histone markers in selected gene in LNCap cells

\begin{tabular}{llllll}
\hline & KCNN2 & PPFIBP2 & JAG1 & SPRED1 & NDRG1 \\
\hline AN-H-AR & 4 & 9 & 1 & 1 & 0 \\
AN-H-HIF & 0 & 4 & 0 & 0 & 2 \\
AN-H-ME1 & 13 & 25 & 2 & 2 & 6 \\
AN-H-ME3 & 0 & 2 & 1 & 1 & 2 \\
AN-N-AR & 8 & 11 & 2 & 6 & 3 \\
AN-N-HIF & 1 & 0 & 0 & 0 & 0 \\
AN-N-ME1 & 18 & 15 & 1 & 6 & 4 \\
AN-N-ME3 & 1 & 3 & 1 & 2 & 1 \\
ET-H-AR & 1 & 7 & 1 & 0 & 0 \\
ET-H-HIF & 0 & 0 & 0 & 0 & 0 \\
ET-H-ME1 & 15 & 19 & 2 & 0 & 5 \\
ET-H-ME3 & 4 & 8 & 2 & 1 & 4 \\
ET-N-AR & 6 & 9 & 1 & 3 & 1 \\
ET-N-HIF & 0 & 0 & 0 & 0 & 0 \\
ET-N-ME1 & 18 & 11 & 2 & 2 & 5 \\
ET-N-ME3 & 4 & 5 & 1 & 1 & 1 \\
\hline AN synhetic & 18 & & & 1 &
\end{tabular}

AN, synthetic androgen (R1881) treatment; ET, ethanol vehicle control; $\mathrm{N}$, normoxia; $\mathrm{H}$, hypoxia; $\mathrm{AR}=$ androgen receptor; $\mathrm{ME1}, \mathrm{H} 3 \mathrm{~K} 4 \mathrm{me}$ 1; ME3, H3K4me3 increased cell growth in the absence and presence of the synthetic androgen R1881, and promoted resistance to ADT in vitro and in vivo. Hypoxia and HIF have already been implicated in the development and progression of CRPC $[34,35]$. Hypoxia was shown to induce AR independence and confer resistance to ADT through a metabolic switch favoring glycolysis [18]. Pseudohypoxia has also been linked to the metabolic switch from oxidative phosphorylation to glycolysis [36]. Expression of HIF1a in normoxia has been reported in androgen dependent prostate cells and in this study we report expression of HIF1a in cells resistant to ADT (LNCaP-Bic, LNCaP$\mathrm{OHF}$ ) and in the androgen independent PC3 cell line ${ }^{1022}$. This study adds to the evidence implicating hypoxia and HIFla in androgen independence, CRPC and ADT resistance.

The high expression of HIF1a in CRPC further supports the role of HIFla in aggressive, androgen dependent prostate cancer. Whether the high expression of HIF1a was associated with pseudohypoxia or hypoxia could not be determined in this study. In future studies the hypoxia marker pimonidazole alongside HIF1a would provide a valuable insight into the contribution of hypoxia and pseudohypoxia in CRPC. 
Table 3 Prognostic significance of selected genes in prostate cancer cohorts

\begin{tabular}{|c|c|c|c|c|c|}
\hline & Swedish & Cambridge & Long & Taylor & TCGA \\
\hline TWIST1 & $\begin{array}{l}2.05 \\
(1.30-3.22) \\
P=0.0019\end{array}$ & $\begin{array}{l}0.95 \\
(0.39-2.35) \\
P=0.917\end{array}$ & $\begin{array}{l}1.46 \\
(0.84-2.54) \\
P=0.178\end{array}$ & $\begin{array}{l}2.69 \\
(1.11-6.49) \\
P=0.028\end{array}$ & $\begin{array}{l}1.72 \\
(1.13-2.60) \\
P=0.01\end{array}$ \\
\hline KCNN2 & $\begin{array}{l}2.39 \\
(1.51-3.79) \\
P=0.000216\end{array}$ & $\begin{array}{l}1.91 \\
(0.75-4.86) \\
P=0.17\end{array}$ & $\begin{array}{l}1.52 \\
(0.87-2.27) \\
P=0.14\end{array}$ & $\begin{array}{l}1.78 \\
(0.78-4.08) \\
P=0.17\end{array}$ & $\begin{array}{l}0.84 \\
(0.56-1.26) \\
P=0.41\end{array}$ \\
\hline SPRED1 & NA & $\begin{array}{l}0.90 \\
(0.36-2.23) \\
P=0.81\end{array}$ & $\begin{array}{l}0.76 \\
(0.44-1.32) \\
P=0.33\end{array}$ & $\begin{array}{l}0.96 \\
(0.43-2.13) \\
P=0.91\end{array}$ & $\begin{array}{l}0.96 \\
(0.64-1.44) \\
P=0.85\end{array}$ \\
\hline PPFIBP2 & NA & $\begin{array}{l}1.21 \\
(0.49-3.02) \\
P=0.68\end{array}$ & $\begin{array}{l}1.73 \\
(0.99-3.03) \\
P=0.05\end{array}$ & $\begin{array}{l}0.86 \\
(0.38-1.92) \\
P=0.70\end{array}$ & $\begin{array}{l}0.76 \\
(0.50-1.13) \\
P=0.17\end{array}$ \\
\hline$J A G 1$ & $\begin{array}{l}1.36 \\
(0.88-2.10) \\
P=0.17\end{array}$ & $\begin{array}{l}2.23 \\
(0.85-5.88) \\
P=0.10\end{array}$ & $\begin{array}{l}2.293 \\
(1.28-4.12) \\
P=0.005\end{array}$ & $\begin{array}{l}1.25 \\
(0.56-2.80) \\
P=0.59\end{array}$ & $\begin{array}{l}1.30 \\
(0.87-1.94) \\
P=0.21\end{array}$ \\
\hline IGFBP3 & $\begin{array}{l}1.00 \\
(0.65-1.55) \\
P=0.99\end{array}$ & $\begin{array}{l}1.09 \\
(0.44-2.68) \\
P=0.86\end{array}$ & $\begin{array}{l}1.56 \\
(0.89-2.73) \\
P=0.12\end{array}$ & $\begin{array}{l}1.99 \\
(0.85-4.66) \\
P=0.11\end{array}$ & $\begin{array}{l}2.07 \\
(1.36-3.20) \\
P=0.0008\end{array}$ \\
\hline NDRG1 & $\begin{array}{l}2.28 \\
(1.43-3.64) \\
P=0.0005\end{array}$ & $\begin{array}{l}1.43 \\
(0.58-3.56) \\
P=0.44\end{array}$ & $\begin{array}{l}0.81 \\
(0.47-1.41) \\
P=0.45\end{array}$ & $\begin{array}{l}0.78 \\
(0.34-1.75) \\
P=0.54\end{array}$ & $\begin{array}{l}0.96 \\
(0.64-1.43) \\
P=0.82\end{array}$ \\
\hline
\end{tabular}

$B C R$ biochemical recurrence; OS overall survival; $N A$ not applicable

Values are hazard ratios ( $95 \%$ confidence intervals). Cohorts were stratified by the median expression of each gene

Gene expression analysis showed few genes were regulated in common by AR, hypoxia, and HIF1a. The finding suggests the signaling pathways act independently and regulate the expression of different subsets of genes. Other studies have reported both positive and negative crosstalk between androgen/AR and hypoxia/HIFla [18, 37, 38]. Globally there were substantially more AR binding sites than HIF binding sites, demonstrating androgen signaling dominance over HIF signaling in the prostate cancer cells studied. Interestingly, hypoxia decreased the number of AR binding sites. This observation contrasts with studies showing hypoxia enhances AR activity [38-40]. The variability in concentration and duration of androgen treatment and hypoxia across studies is most likely responsible for the conflicting results. The observed decrease in androgen binding sites under hypoxia in our study may be explained by conformational changes in chromatin structure induced by $12 \mathrm{~h}$ exposure to $1 \%$ hypoxia, which may restrict the accessibility of AR binding sites [41].

The locations of the AR DNA binding sites in the ethanol vehicle control and R1881 treated cells were highly conserved. The 2-fold increase in AR binding sites with R1881 treatment added to the existing AR binding sites that were occupied in the absence of R1881. In comparison, the DNA binding sites occupied by HIF under normoxia were located in different regions to the HIF binding sites occupied under hypoxia, indicating that HIF binds to different sites in the DNA and promotes the expression of a different subset of genes under pseudohypoxia and hypoxia. Histone marks associated with active transcription were globally decreased within the DNA following synthetic androgen R1881 treatment. In contrast hypoxia marginally increased the presence the two histone marks, and it has previously been reported that hypoxia rapidly increases histone methylation independently of HIF [42]. Despite decreasing the prevalence of H3K4me3, the location of this histone mark within promoter regions was increased as a result of R1881 treatment and indicates enhanced transcriptional activity.

We found few HIF transcription factor binding sites within the introns and exons of the seven genes upregulated by androgen and HIF1a suggesting the HIF regulated expression of these genes is most likely driven by it binding to distal sites [43]. The greatest number of AR binding sites within the genes was observed with androgen treatment under normoxia, with a reduction in the number of AR binding sites under hypoxia. This decrease in AR binding sites under hypoxia was observed globally, possibly as a result of hypoxia induced conformational changes in the DNA which restrict the accessibility of AR binding sites. This implies that the functional relationship between the AR and HIF1a cannot be defined based on physical interaction and colocalization of these transcription factors at particular binding sites in the genome, in contrast to some studies on ETS factors. Instead there are potentially higher order changes in chromatin conformation that could permit independent distal sites occupied by these two transcription factors to interact and perhaps interfere 
with each other. An in-depth exploration of this is beyond the scope of this study and will require chromosome conformation capture methods coupled to highthroughput sequencing. It also is possible that many of the HIFla effects on AR binding are much more indirect than this and reflect changes in i) intracellular signaling and metabolism ii) concomitant epigenetic changes for example in DNA methylation iii) changes in the expression and of other transcription factors which collectively impact on AR recruitment and gene regulation. In-depth dissection of each of these aspects awaits future studies however it is intriguing that amongst the small number of genes upregulated by both androgen and HIF one, TWIST1, is a transcription factor and was the most prognostic.

Upregulated TWIST1 and AR expression have previously been reported in a castration resistant LNCaP mouse model, implicating crosstalk between epithelial mesenchymal transition and castration resistance [44]. TWIST1 was also shown to upregulate AR expression and to be upregulated in response to ADT [45]. The variability in prognostic significance between the cohorts may in part be due to use of different gene expression platforms. A further limitation is that most patients in the cohorts had primary prostate cancer treated by radical prostatectomy without hormone therapy and were mostly low and intermediate risk patients. Considering the seven genes identified in this study are upregulated by androgen, HIF1a and/or hypoxia it is hypothesized that they promote disease progression and development of CRPC and it would be interesting to look at the expression of these genes in high risk and advanced prostate cancer cohorts. As AR and HIF signaling axes are active in CRPC these seven genes are potential biomarkers of aggressive disease that might be useful to predict likely disease progression towards CRPC $[46,47]$.

\section{Conclusion}

In this study the absence of HIF1a and endogenous androgen in vivo resulted in regression of tumor growth but HIF1a signaling could restore tumor growth in the absence of AR signaling. The data presented here indicate simultaneous therapeutic inhibition of the HIF1a and AR signaling pathways is a potential therapeutic strategy, as has previously been proposed [47]. We show that the oncogenic signaling pathways target the expression of different subsets of genes but both promote proliferation, tumor growth and disease progression. The relationship between the AR and HIFla signaling pathways and their association with the development of CRPC could be exploited to identify predictive biomarkers of progression to CRPC and dual targeting of the AR and hypoxia/HIF1a should be further investigated for patients most at risk of developing CRPC.

\section{Supplementary information}

Supplementary information accompanies this paper at https://doi.org/10. 1186/s12885-020-06890-6.

\section{Additional file 1.}

\section{Abbreviations}

ADT: Androgen deprivation therapy; AR: Androgen receptor; BCR: Biochemical recurrence free; Bic: Bicalutamide; ChIP: Chromatin immunoprecipitation; CRPC: Castrate resistant prostate cancer; HIF1a: Hypoxia inducible factor 1a; PSA: Prostate specific antigen; OHF: Hydroxyflutamide

\section{Acknowledgements}

We acknowledge members of the Uro-Oncology laboratory for helpful discussions. We thank research staff of Cancer Research UK Cambridge Institute core facilities for technical support.

\section{Authors' contributions}

DEN, IGM and MT conceived the project and experimental design. DEN and IGM supervised the work. $\mathrm{MO}$ and $\mathrm{JH}$ undertook the sequencing and microarray profiling. TC and RS performed computational analyses on the sequencing data. LY evaluated the prognostic potential of transcripts and undertook integrative analyses. MT and FL performed the experiments. MT, FL and BASB and analysed the data. HS, MT, DS and AW performed the immunohistochemistry and scoring. ARM and MT performed the in vivo experiments. BASB, MT, IGM, DEN, LY, CMLW, PM and FL drafted and revised the manuscript. All authors have read and approved the manuscript.

\section{Funding}

This study was supported by the National Cancer Research Institute (National Institute of Health Research (NIHR) Collaborative Study: 'Prostate Cancer: Mechanisms of Progression and Treatment (PROMPT)" (grant G0500966/ 75466) and the Academy of Medical Sciences (Clinical Lecturer starter grant). We thank the National Institute for Health Research, Hutchison Whampoa Limited, the Human Research Tissue Bank (Addenbrooke's Hospital), Cancer Research UK, and the NIHR Manchester Biomedical Centre. The tissue samples used for the immunohistochemistry were collected with the aforementioned support of the PROMPT and NIHR. The experimental/ laboratory work was funded by a Cancer Research UK program grant (DEN). CMLW, BASB, IGM and LY are members of a Prostate Cancer UK/Movember Centre of Excellence (CEO13_2-004) which supported further analysis of the data and manuscript preparation and IGM was also supported in this regard the John Black Charitable Foundation.

\section{Availability of data and materials}

Microarray and ChlP-seq data generated have been deposited within the $\mathrm{Na}$ tional Center for Biotechnology Information (NCB) Gene Expression Omnibus (GEO) database (https://www.ncbi.nlm.nih.gov/geo/) under GSE114734.

\section{Ethics approval and consent to participate}

Clinical samples were collected from Cambridge University Hospitals NHS Trust as part of the PROMPT study, and ethical approval was granted by the local research and ethics committee (LREC number: 02/281 M) and by the multicenter research and ethics committee (MREC number 01/4061). Patients were enrolled into these studies under this ethical framework (PROMPT https://gtr.ukri.org/project/F3045258-1D21-4189-BD51-B1D15232AF6D) which involved obtaining informed written consent. Samples collected through this framework are eligible for use in a range of translation prostate cancer research projects and underpin a number of past and current research projects.

\section{Consent for publication}

Not applicable.

\section{Competing interests}

The authors declare that they have no competing interest.

\section{Author details}

'Uro-oncology Research Group, Cancer Research UK Cambridge Institute, Cambridge CBO2 ORE, UK. ${ }^{2}$ UCL division of Surgery and Interventional 
Science, Royal Free Hospital, Pond Street, London NW3 2QG, UK. ${ }^{3}$ Division of Cancer Sciences, School of Medical Sciences, Faculty of Biology, Medicine and Health, University of Manchester, Manchester Academic Health Science Centre, Christie Hospital NHS Trust, M20 4BX, Manchester, UK. ${ }^{4}$ Department of Pathology, Addenbrooke's Cambridge University Hospital, Cambridge, UK. ${ }^{5}$ Division of Medicine, University College London, London, UK. ${ }^{6}$ Department of Oncology, University of Cambridge, London CB2 OXZ, UK. ${ }^{7}$ Cambridge Institute of Medical Research, Cambridge Biomedical Campus, Cambridge CB2 0SP, UK. ${ }^{8}$ Manchester Biomedical Research Centre, University of Manchester, Central Manchester University Hospitals NHS Foundation Trust, Manchester, UK. ${ }^{~}$ Patrick G Johnston Centre for Cancer Research and Cell Biology, Queens University Belfast, Belfast BT9 7AE, UK. ${ }^{10}$ Nuffield Department of Surgical Sciences, University of Oxford, Oxford OX3 9DU, UK. ${ }^{11}$ Academic Urology Group, University of Cambridge, Cambridge, UK.

Received: 6 February 2020 Accepted: 22 April 2020

Published online: 25 May 2020

\section{References}

1. Massie CE, Lynch A, Ramos-Montoya A, Boren J, Stark R, Fazli L, et al. The androgen receptor fuels prostate cancer by regulating central metabolism and biosynthesis. EMBO J. 2011;30(13):2719-33.

2. Meng MV, Grossfeld GD, Sadetsky N, Mehta SS, Lubeck DP, Carroll PR. Contemporary patterns of androgen deprivation therapy use for newly diagnosed prostate cancer. Urology. 2002;60(3 Suppl 1):7-11 discussion -2.

3. Kirby M, Hirst C, Crawford ED. Characterising the castration-resistant prostate cancer population: a systematic review. Int J Clin Pract. 2011;65(11):1180-92.

4. Mills IG. Maintaining and reprogramming genomic androgen receptor activity in prostate cancer. Nat Rev Cancer. 2014;14(3):187-98.

5. Visakorpi T, Hyytinen E, Koivisto P, Tanner M, Keinanen R, Palmberg C, et al. In vivo amplification of the androgen receptor gene and progression of human prostate cancer. Nat Genet. 1995;9(4):401-6.

6. Antonarakis ES, Armstrong AJ, Dehm SM, Luo J. Androgen receptor variantdriven prostate cancer: clinical implications and therapeutic targeting. Prostate Cancer Prostatic Dis. 2016;19(3):231-41.

7. McCall P, Gemmell LK, Mukherjee R, Bartlett JM, Edwards J. Phosphorylation of the androgen receptor is associated with reduced survival in hormonerefractory prostate cancer patients. Br J Cancer. 2008;98(6):1094-101.

8. Massie CE, Adryan B, Barbosa-Morais NL, Lynch AG, Tran MG, Neal DE, et al. New androgen receptor genomic targets show an interaction with the ETS1 transcription factor. EMBO Rep. 2007;8(9):871-8.

9. Sharma NL, Massie CE, Butter F, Mann M, Bon H, Ramos-Montoya A, et al. The ETS family member GABPalpha modulates androgen receptor signalling and mediates an aggressive phenotype in prostate cancer. Nucleic Acids Res. 2014:42(10):6256-69.

10. Baena E, Shao Z, Linn DE, Glass K, Hamblen MJ, Fujiwara Y, et al. ETV1 directs androgen metabolism and confers aggressive prostate cancer in targeted mice and patients. Genes Dev. 2013;27(6):683-98.

11. Chen $Y$, Sawyers CL. Coordinate transcriptional regulation by ERG and androgen receptor in fusion-positive prostate cancers. Cancer Cell. 2010;17(5):415-6.

12. Yu J, Yu J, Mani RS, Cao Q, Brenner CJ, Cao X, et al. An integrated network of androgen receptor, polycomb, and TMPRSS2-ERG gene fusions in prostate cancer progression. Cancer Cell. 2010;17(5):443-54.

13. Watson PA, Arora VK, Sawyers CL. Emerging mechanisms of resistance to androgen receptor inhibitors in prostate cancer. Nat Rev Cancer. 2015; 15(12):701-11.

14. Williamson JR, Chang K, Frangos M, Hasan KS, Ido Y, Kawamura T, et al. Hyperglycemic pseudohypoxia and diabetic complications. Diabetes. 1993; 42(6):801-13.

15. Hayashi Y, Yokota A, Harada H, Huang G. Hypoxia/pseudohypoxia-mediated activation of hypoxia-inducible factor-1a in cancer. Cancer Sci. 2019;110(5): 1510-7.

16. Vergis R, Corbishley CM, Norman AR, Bartlett J, Jhavar S, Borre M, et al. Intrinsic markers of tumour hypoxia and angiogenesis in localised prostate cancer and outcome of radical treatment: a retrospective analysis of two randomised radiotherapy trials and one surgical cohort study. Lancet Oncol. 2008;9(4):342-51.

17. Huang $M$, Du $H$, Zhang $L$, Che $H$, Liang $C$. The association of HIF-1a expression with clinicopathological significance in prostate cancer: a metaanalysis. Cancer Manag Res. 2018;10:2809-16.
18. Geng H, Xue C, Mendonca J, Sun XX, Liu Q, Reardon PN, et al. Interplay between hypoxia and androgen controls a metabolic switch conferring resistance to androgen/AR-targeted therapy. Nat Commun. 2018;9(1):4972.

19. Horii K, Suzuki Y, Kondo Y, Akimoto M, Nishimura T, Yamabe Y, et al. Androgen-dependent gene expression of prostate-specific antigen is enhanced synergistically by hypoxia in human prostate cancer cells. Mol Cancer Res. 2007;5(4):383-91.

20. Tong D, Liu Q, Liu G, Yuan W, Wang L, Guo Y, et al. The HIF/PHF8/AR axis promotes prostate cancer progression. Oncogenesis. 2016;5(12):e283.

21. Lunardi P, Beauval JB, Roumiguie M, Soulie M, Cuvillier O, Malavaud B. Mechanisms of castration resistance: Intratumoral hypoxia stimulates the androgen receptor expression. Prog Urol. 2016;26(3):159-67.

22. Lynch AG, Hadfield J, Dunning MJ, Osborne M, Thorne NP, Tavare S. The cost of reducing starting RNA quantity for Illumina BeadArrays: a bead-level dilution experiment. BMC Genomics. 2010;11:540.

23. Schmidt D, Stark R, Wilson MD, Brown GD, Odom DT. Genome-scale validation of deep-sequencing libraries. PLoS One. 2008;3(11):e3713.

24. Zhu LJ, Gazin C, Lawson ND, Pages H, Lin SM, Lapointe DS, et al. ChIPpeakAnno: a bioconductor package to annotate ChIP-seg and ChIPchip data. BMC Bioinformatics. 2010;11:237.

25. Ross-Adams H, Lamb AD, Dunning M, Halim S, Lindberg J, Massie CM, et al. Integration of copy number and transcriptomics provides risk stratification in prostate cancer: a discovery and validation cohort study. EBioMedicine. 2015;2(9):1133-44.

26. Taylor BS, Schultz N, Hieronymus H, Gopalan A, Xiao Y, Carver BS, et al. Integrative genomic profiling of human prostate cancer. Cancer Cell. 2010;18(1):11-22.

27. Long Q, Xu J, Osunkoya AO, Sannigrahi S, Johnson BA, Zhou W, et al. Global transcriptome analysis of formalin-fixed prostate cancer specimens identifies biomarkers of disease recurrence. Cancer Res. 2014;74(12):3228-37.

28. Sboner A, Demichelis F, Calza S, Pawitan Y, Setlur SR, Hoshida Y, et al. Molecular sampling of prostate cancer: a dilemma for predicting disease progression. BMC Med Genet. 2010;3:8.

29. Huang X, Gao X, Li W, Jiang S, Li R, Hong H, et al. Stable H3K4me3 is associated with transcription initiation during early embryo development. Bioinformatics. 2019.

30. Rada-Iglesias A. Is H3K4me1 at enhancers correlative or causative? Nat Genet. 2018:50(1):4-5.

31. Zeitz MJ, Ay F, Heidmann JD, Lerner PL, Noble WS, Steelman BN, et al. Genomic interaction profiles in breast cancer reveal altered chromatin architecture. PLoS One. 2013;8(9):e73974.

32. Yang L, Roberts D, Takhar M, Erho N, Bibby BAS, Thiruthaneeswaran N, et al. Development and validation of a 28-gene hypoxia-related prognostic signature for localized prostate Cancer. EBioMedicine. 2018;31:182-9.

33. Ranasinghe WK, Baldwin GS, Bolton D, Shulkes A, Ischia J, Patel O. HIF1alpha expression under normoxia in prostate cancer--which pathways to target? J Urol. 2015;193(3):763-70.

34. Ranasinghe WK, Xiao L, Kovac S, Chang M, Michiels C, Bolton D, et al. The role of hypoxia-inducible factor 1alpha in determining the properties of castrate-resistant prostate cancers. PLoS One. 2013;8(1):e54251.

35. Milosevic M, Warde $P$, Ménard C, Chung P, Toi A, Ishkanian A, et al. Tumor hypoxia predicts biochemical failure following radiotherapy for clinically localized prostate cancer. Clin Cancer Res. 2012;18(7):2108-14.

36. Zecchini V, Madhu B, Russell R, Pertega-Gomes N, Warren A, Gaude E, et al. Nuclear ARRB1 induces pseudohypoxia and cellular metabolism reprogramming in prostate cancer. EMBO J. 2014;33(12):1365-82.

37. Mabjeesh NJ, Willard MT, Frederickson CE, Zhong H, Simons JW. Androgens stimulate hypoxia-inducible factor 1 activation via autocrine loop of tyrosine kinase receptor/phosphatidylinositol 3'-kinase/protein kinase B in prostate cancer cells. Clin Cancer Res. 2003;9(7):2416-25.

38. Park SY, Kim YJ, Gao AC, Mohler UL, Onate SA, Hidalgo AA, et al. Hypoxia increases androgen receptor activity in prostate cancer cells. Cancer Res. 2006;66(10):5121-9.

39. Mitani T, Yamaji R, Higashimura Y, Harada N, Nakano Y, Inui H. Hypoxia enhances transcriptional activity of androgen receptor through hypoxiainducible factor-1alpha in a low androgen environment. J Steroid Biochem Mol Biol. 2011;123(1-2):58-64.

40. Park C, Kim Y, Shim M, Lee Y. Hypoxia enhances ligand-occupied androgen receptor activity. Biochem Biophys Res Commun. 2012;418(2):319-23.

41. Johnson $A B$, Denko N, Barton MC. Hypoxia induces a novel signature of chromatin modifications and global repression of transcription. Mutat Res. 2008:640(1-2):174-9.

42. Batie M, Frost J, Frost M, Wilson JW, Schofield P, Rocha S. Hypoxia induces rapid changes to histone methylation and reprograms chromatin. Science. 2019;363(6432):1222-6. 
43. Schodel J, Oikonomopoulos S, Ragoussis J, Pugh CW, Ratcliffe PJ, Mole DR. High-resolution genome-wide mapping of HIF-binding sites by ChIP-seq. Blood. 2011;117(23):e207-17.

44. Shiota M, Itsumi M, Takeuchi A, Imada K, Yokomizo A, Kuruma H, et al. Crosstalk between epithelial-mesenchymal transition and castration resistance mediated by Twist1/AR signaling in prostate cancer. Endocr Relat Cancer. 2015;22(6):889-900.

45. Shiota M, Yokomizo A, Tada Y, Inokuchi J, Kashiwagi E, Masubuchi D, et al. Castration resistance of prostate cancer cells caused by castration-induced oxidative stress through Twist1 and androgen receptor overexpression. Oncogene. 2010;29(2):237-50.

46. Coutinho I, Day TK, Tilley WD, Selth LA. Androgen receptor signaling in castration-resistant prostate cancer: a lesson in persistence. Endocr Relat Cancer. 2016;23(12):T179-T97.

47. Fernandez EV, Reece KM, Ley AM, Troutman SM, Sissung TM, Price DK, et al. Dual targeting of the androgen receptor and hypoxia-inducible factor 1alpha pathways synergistically inhibits castration-resistant prostate cancer cells. Mol Pharmacol. 2015;87(6):1006-12.

\section{Publisher's Note}

Springer Nature remains neutral with regard to jurisdictional claims in published maps and institutional affiliations.

Ready to submit your research? Choose BMC and benefit from:

- fast, convenient online submission

- thorough peer review by experienced researchers in your field

- rapid publication on acceptance

- support for research data, including large and complex data types

- gold Open Access which fosters wider collaboration and increased citations

- maximum visibility for your research: over $100 \mathrm{M}$ website views per year

At $\mathrm{BMC}$, research is always in progress.

Learn more biomedcentral.com/submissions 